\title{
Australian Journal of

\section{Chemical composition of garlic wood (Gallesia integrifolia) (Phytolaccaceae) volatile compounds and their activity on cattle tick}

\author{
Keila Fernanda Raimundo ${ }^{1,3}$, Wanessa de Campos Bortolucci ${ }^{1}$, Eloísa Schneider Silva ${ }^{2}$, Ana Flávia \\ Balisk Pereira ${ }^{3}$, Otávio Akira Sakai ${ }^{3}$, Ranulfo Piau Júnior ${ }^{4}$, José Eduardo Gonçalves ${ }^{5,6}$, Giani Andréa \\ Linde $^{1}$ and Zilda Cristiani Gazim ${ }^{1}$
}

\author{
${ }^{1}$ Programa de Pós-Graduação em Biotecnologia Aplicada à Agricultura. Universidade Paranaense - Unipar, \\ Umuarama-PR, Brazil \\ ${ }^{2}$ Curso de Graduação em Farmácia. Universidade Paranaense - Unipar, Umuarama-PR, Brazil \\ ${ }^{3}$ Instituto Federal do Paraná - IFPR, Campus Umuarama-PR, Brazil \\ ${ }^{4}$ Programa de Pós-Graduação em Ciência Animal da Universidade Paranaense - Unipar, Umuarama-PR, Brazil \\ ${ }^{5}$ Mestrado em Tecnologia Limpas e Mestrado em Promoção da Saúde, UniCesumar, Maringá-PR, Brazil \\ ${ }^{6}$ Instituto Cesumar de Ciência, Tecnologia e Inovação - ICETI, Maringá-PR, Brazil
}

\section{*Corresponding author: cristianigazim@unipar.br}

\begin{abstract}
The objective of this study was to evaluate the acaricidal and larvicidal activities of essential oil (EO) from fruits, leaves and flowers of garlic wood on cattle tick [Rhipichephalus (Boophilus) microplus]. The fruits were harvested from May to June (2015) and the leaves and flowers in December (2015). The EO was obtained by hydrodistillation (2h) and identified by GC/MS. Bioassays consisted of Adult Immersion Test and Larval Immersion Test. The results made the following major compounds evident: 2,3,5trithiahexane $(35.29 \%)$ in fruits, dimethyl sulfide $(42.42 \%)$ in leaves and methanethiol $(44.91 \%)$ in flowers. The EOs from fruits, leaves and flowers showed high activity on the tick larval cycle, presenting $\mathrm{LD}_{99.9}$ of $(0.23 \pm 0.01 \mathrm{mg} / \mathrm{mL}),(2.15 \pm 0.11 \mathrm{mg} / \mathrm{mL}) \mathrm{and}$ $(0.08 \pm 0.00 \mathrm{mg} / \mathrm{mL})$, respectively. However, when we compared different phases of cattle tick's live cycle, EO from fruits was more active on females' mortality, and EO from leaves was more efficient on the egg hatching inhibition, whereas EO from flowers presented better results on bovine tick larva. Thus, garlic wood (Gallesia integrifolia), a native plant of the Atlantic forest can be considered as a promising natural agent to control bovine tick.
\end{abstract}

Keywords: Garlic wood, 2,3,5-trithiahexane, dimethyl sulfide, methanethiol, Rhipichephalus (Boophilus) microplus, dynamic headspace.

Abbreviations: GC/MS_gas chromatographer coupled to mass spectrometer; LD_lethal dose; LD 99.9 lethal dose to eliminate $99.9 \%$ of larvae and ticks, OE_essential oil.

\section{Introduction}

Brazil has the greatest commercial cattle herd in the world with more than 200 million heads. However, the productivity could be greater if it were not affected by Rhipichephalus (Boophilus) microplus, which causes big losses to livestock (Cerri et al., 2015). Cattle is this parasite's main host and the principal economic losses are related to the low feed to gain ratio, chronic loss, reduction of milk and beef production, decrease in leather quality, skin lesions that favor the development of myiases, anemia and transmission of pathogens, leading to the animal's death (Alvaréz et al., 2008). One of the strategies to minimize these losses involves the reduction of tick populations to economically acceptable levels (Martins et al., 2002). Tick control is based on the utilization of chemical products such as pyrethroids, organophosphates and avermectins. However, the excessive use of these products has led to the development of ticks resistant to these acaricides (Kafle et al., 2012). Moreover, commercial acaricides are toxic to human and can contaminate the environment and leave residues in beef and milk once they are generally sprayed or injected in the animals (Furtado et al., 2013). In order to minimize these problems, an alternative to substitute these chemical products is the use of natural products such as extracts and essential oils (EO) from plants, which are becoming quite promising, contributing to intensify research studies and technologies that promote sustainable development (Souza et al., 2010). In this context, the Atlantic Forest is considered as one of the greatest biodiversity centers worldwide, which presents a great variety of aromatic and medicinal species, and among them garlic wood (pau d'alho in Portuguese). It is a native plant of South America that is found in Peru and Brazil, from Ceará to Paraná (Sambuichi, 2009) which stands out due to its broad application in popular medicine due to its alliaceous characteristic (Lorenzi, 2002).

Botanically, garlic wood is called Gallesia integrifolia (SPRENG.) Harms, because it was first described by Sprengel in 1821, and named Thouinia integrifolia Spreng.; however, this species presents scientific synonym's such as Gallesia gorarema, Gallesia gorazema (Vell.) Moq., Crataeva gorazema Vell. (Akisue et al., 1986). This large 
species belongs to Phytolaccaceae family and when it is green, it characteristically exhales a strong alliaceous smell which is peculiar compared to all other plants (Duringan et al., 1997). The plant leaves are elliptical and shiny, flowers are bunchy and white which bloom from February to April. Its elongated samara-like fruits become brownish when ripening from June to October; and it is difficult to separate the seed from the fruit (Akisue et al., 1986; Duringan et al., 1997). Gallesia integrifolia also produces wood for several uses. It has characteristic smell of garlic when green and, therefore, it is popularly named garlic wood. The smell vanishes when the wood gets dry, and then the wood becomes durable and is used as a substitute of pine (Maia et al., 2013).

Thus, this study aimed to evaluate the acaricidal and larvicidal activity of garlic wood EO from fruits, leaves and flowers on $R$. (B.) microplus and to determine the chemical composition of in natura vegetal material by dynamic headspace technique.

\section{Results}

\section{Physical and chemical indices}

The results obtained for the refraction index and the relative density of garlic wood EO from fruits, leaves and flowers are shown in Table 1.

\section{Chemical composition of garlic wood essential oil}

The chemical identification was done by GC/MS using dynamic headspace technique. In natura fruits of garlic wood were utilized in chemical analysis and 44 compounds were eluted and 43 were identified (Table 2). Among them, the major compounds are: methanethiol (16.26\%), dimethyl sulfone $(9.08 \%), \quad 2,3,5$-trithiahexane $(35.29 \%), \quad 2,3,5,6$ tetrathiaheptane $(5.22 \%)$ and 3,6-dithiaoctan-1,8-diol (20.89\%) (Fig 1).

In the chemical identification of in natura garlic wood leaves, 44 compounds were identified (Table 3 ), and the ones that stood out as major compounds were: dimethyl sulfide (42.42\%), 3-methylbutanal (40.43\%), ethanol, 2-(methylthio) $(5.52 \%)$ and $\alpha$-terpinolene (8.63\%) (Fig 2).

The chemical identification was also done for in natura garlic wood flowers which represented 44 compounds (Table 4). The major compounds were: methanethiol (44.91\%), dimethyl sulfide (43.72\%) and 2-butanamine (4.70\%) (Fig 3).

\section{Acaricidal and larvicidal activity on Rhipichephalus (Boophilus) microplus}

The results obtained for acaricidal activity of garlic wood EO from fruits, leaves and flowers on $R$. (B) microplus are shown on Tables 5, 6 and 7, respectively.

The inhibition of egg hatching is one of the relevant parameters to measure the acaricidal activity of Eos. This parameter allows acaricide control. Evaluation of garlic wood EOs from fruits, leaves and flowers verified that EO from leaves presented greater egg hatching inhibition $(56.99 \%)$ at the concentration of $10.00 \mathrm{mg} / \mathrm{mL}$ (Table 6).

According to the Ministry of Agriculture (Brasil, 1990), for a product to be considered efficient, the value determined for the product efficiency (\%) should be $95.00 \%$ minimum. Thus, garlic wood EO from fruits, leaves and flowers presented product efficiency (93.97, 93.97 and 98.67\%), respectively, at the concentration of $100.00 \mathrm{mg} / \mathrm{mL}$.
The results of lethal doses (LDs) of garlic wood EO from fruits, leaves and flowers needed to eliminate $50 \%\left(\mathrm{LD}_{50}\right)$ and $99.9 \%\left(\mathrm{LD}_{99.9}\right)$ of ingurgitated female ticks and larvae (Table 8).

Evaluation of garlic wood EO from fruits, leaves and flowers on female mortality verified that EO from fruits had greater action with $\mathrm{LD}_{99.9}(251.59 \mathrm{mg} / \mathrm{mL})$. However, the evaluation of the effect of garlic wood'd EO from fruits, leaves and flowers on tick larvae showed that EO from flowers provided greater larvicidal activity with $\mathrm{LD}_{99.9}(0.08 \mathrm{mg} / \mathrm{mL})$.

\section{Discussion}

\section{Physical and chemical indices}

Analysis of relative density of garlic wood EO from fruits, leaves and flowers verified that there was no significant difference among the results which presented values with greater density than water $(\mathrm{d}=0.99 \mathrm{~g} / \mathrm{mL}$ at the temperature of $20{ }^{\circ} \mathrm{C}$ ) (Constantino et al., 2004)(Table 1). These results are in accordance with those observed during the hydrodistillation procedures of garlic wood EO from fruits, leaves and flowers when the EO was accumulated in the bottom end of the condenser, which meant it is denser than water. The refraction index is defined as the relation between the light speed in the vacuum and its speed in the substance, and is one of the physical and chemical parameters that characterizes EO of a specific species (Farmacopeia, 1988; Simões et al., 2010). In that parameter, the EO from fruits, leaves and flowers did not present significant difference as well (Table 1). The evaluation of physical and chemical indices of EO is considered fundamental for the industry since the parameters for the EO quality are considered. Moreover, the refraction index and the relative density allow the detection of EO adulterations (Gil, 2007).

\section{Analysis of chemical compounds by dynamic headspace}

The presence of natural sulfur products is a chemotaxonomic characteristic of the species of Phytolacacceae family. These compounds, volatile organophosphates also present in onions (Schünemann et al., 2006; De Souza et al., 2015) are related to the characteristic odor of garlic and are formed by the action of alinase enzymes (Block, 1992). The presence of these sulfur compounds was identified in the chemical composition obtained by dynamic headspace of fruits, leaves and fresh flowers of garlic wood (Tables 1, 2 and 3). It was also verified in garlic wood EO from fruits, leaves and flowers obtained by hydro distillation.

There are no studies that report the chemical composition obtained by dynamic headspace utilizing fruits, leaves and flowers of in natura garlic wood; however, some of the volatile sulfur compounds found in this study had already been identified.

The vegetal cell is able to biosynthesize a variety of metabolites that are important to the development, adaptation and protection of the plant. These metabolites are utilized in the pharmaceutical, food and biofuel industries. However, some secondary metabolites may have a sulfur atom in their molecule such as the amino acids methionine and cysteine, which provide the synthesis of organosulfur compounds through thermal or enzymatic processes (Kyung and Lee, 2001; Dewick, 2002); therefore, they are able to direct the synthesis of organosulfur compounds.

Therefore, methionine an amino acid found in Phytolaccaceae species (Dewick, 2002), degrades by thermal process and produces methional, which in turn degrades itself 
Table 1. Physical and chemical indices of the essential oil of fruits, leaves and flowers of Gallesia integrifolia.

\begin{tabular}{lcc}
\hline Essential Oil & \multicolumn{2}{c}{ Physical and Chemical Indices } \\
\hline & Refraction Index & Relative Density $(\mathrm{g} / \mathrm{mL})$ \\
& $\boldsymbol{n}_{\boldsymbol{D}}^{\mathbf{2 0}}$ & $\boldsymbol{d}_{\mathbf{2 0}}^{\mathbf{2 0}}$ \\
\hline Fruit & $1.6205^{\mathrm{a}}$ & $1.48^{\mathrm{a}}$ \\
Leaf & $1.6075^{\mathrm{a}}$ & $1.45^{\mathrm{a}}$ \\
Flower & $1.6224^{\mathrm{a}}$ & $1.37^{\mathrm{a}}$ \\
\hline Means followed by the same letter do not differ from each other by the Scott-Knott test $\mathrm{p}(0.05)$.
\end{tabular}

Table 2. Chemical composition obtained from fruits in natura of Gallesia integrifolia by dynamic headspace.

\begin{tabular}{|c|c|c|c|}
\hline Peak & ${ }^{\mathrm{b}}$ Compounds & Area $(\%)$ & $\begin{array}{c}\text { Identification } \\
\text { method }\end{array}$ \\
\hline 1 & Methanethiol & 16.26 & $\mathrm{a}, \mathrm{b}$ \\
\hline 2 & Dimethyl sulfide & 1.42 & $a, b$ \\
\hline 3 & Dimethyl sulfoxide & 0.36 & $a, b$ \\
\hline 4 & 3-methylbutanal & 0.68 & $a, b$ \\
\hline 5 & Dimethyl disulfide & 1.06 & $a, b$ \\
\hline 6 & Dimethyl sulfone & 9.08 & $a, b$ \\
\hline 7 & 2-ethylfuran & 0.45 & $a, b$ \\
\hline 8 & Hexanal & 0.23 & $a, b$ \\
\hline 9 & Santene & $\mathrm{t}$ & $a, b$ \\
\hline 10 & 2,4-dithiapentane & 0.35 & $a, b$ \\
\hline 11 & Artemesia triene & $\mathrm{t}$ & $a, b$ \\
\hline 12 & $\alpha$-pinene & $\mathrm{t}$ & $a, b$ \\
\hline 13 & $\alpha$-fenchene & $\mathrm{t}$ & $a, b$ \\
\hline 14 & Camphene & $\mathrm{t}$ & $a, b$ \\
\hline 15 & Sabinene & $\mathrm{t}$ & $a, b$ \\
\hline 16 & Isolimonene & $\mathrm{t}$ & $a, b$ \\
\hline 17 & Dimethyl thiosulfonate & 3.49 & $a, b$ \\
\hline 18 & Dimethyl trisulfide & 0.22 & $a, b$ \\
\hline 19 & Pentylfuran & 0.33 & $a, b$ \\
\hline 20 & $p$-mentha-1,8-diene & $\mathrm{t}$ & $a, b$ \\
\hline 21 & $\alpha$-terpinene & $\mathrm{t}$ & $a, b$ \\
\hline 22 & $\gamma$-terpinene & $\mathrm{t}$ & $a, b$ \\
\hline 23 & cis-linalool oxide & $\mathrm{t}$ & $a, b$ \\
\hline 24 & trans-linalool oxide & $\mathrm{t}$ & $a, b$ \\
\hline 25 & Terpinolene & $\mathrm{t}$ & $a, b$ \\
\hline 26 & Linalool & 0.33 & $a, b$ \\
\hline 27 & 2,3,5-trithiahexane & 35.29 & $a, b$ \\
\hline 28 & trans-p-menth-2-en-1,8-diol & $\mathrm{t}$ & $a, b$ \\
\hline 29 & $\alpha$-terpineol & $\mathrm{t}$ & $a, b$ \\
\hline 30 & Trithiometoxymethane & 0.39 & $a, b$ \\
\hline 31 & 2,8-dithianonane & $\mathrm{t}$ & $a, b$ \\
\hline 32 & 3,5-dithiahexanol-5,5-dioxide & $\mathrm{t}$ & $a, b$ \\
\hline 33 & $\gamma$-terpineol & $\mathrm{t}$ & $a, b$ \\
\hline 34 & $\alpha$-endo-fenchylacetate & $\mathrm{t}$ & $a, b$ \\
\hline 35 & 2,3,5,6-tetrathiaheptane & 5.22 & $a, b$ \\
\hline 36 & Isobornyl acetate & 0.64 & $a, b$ \\
\hline 37 & L-methionine, ethyl ester & $\mathrm{t}$ & $a, b$ \\
\hline 38 & Thymol & $\mathrm{t}$ & $a, b$ \\
\hline 39 & $\beta$-ionone & $\mathrm{t}$ & $\mathrm{a}, \mathrm{b}$ \\
\hline 40 & 3,6-dithiaoctan-1,8-diol & 20.89 & $a, b$ \\
\hline 41 & n.i. & $\mathrm{t}$ & $a, b$ \\
\hline 42 & N-ethyl-1,3-dithioisoindole & 1.05 & $a, b$ \\
\hline 43 & 5-methyl-2-phenylindole & 0.42 & $a, b$ \\
\hline 44 & $\begin{array}{l}\text { 1,3-dimethyl-4- } \\
\text { azaphenanthrene }\end{array}$ & 1.82 & $\mathrm{a}, \mathrm{b}$ \\
\hline
\end{tabular}

${ }^{\mathrm{a}} \mathrm{MS}$ identification based on comparison of mass spectra using Wiley 275 libraries. ${ }^{\mathrm{b}}$ Compounds listed in order of elution in column HP-5MS. $\mathrm{n} . \mathrm{i} .=$ not identified. $\mathrm{t}=$ traces. Area $(\%)$ : percentage $(\%)$ of the area occupied by compounds within the chromatogram.

(Table 2) and flowers (Table 4) of garlic wood, which can be transformed into dimethyl disulfide in the presence of oxygen (Schutte and Teranishi, 1974). Through a transfer reaction of the methyl group, it can also form dimethyl sulfide (Fig 2 and 3) (Lomans et al., 2002), one of the major compounds in leaves (Table 3) and flowers (Table 4) of garlic wood (Fig 4). Sulfur compounds are found in food and beverages and can have an attractive or repulsive role (Landaud et al., 2009), as methanethiol, a very important compound for the aroma of some cheeses such as cheddar (Burbank and Qian, 2005). On the other hand, it is one of the compounds that contribute to halitosis produced by periodontal bacteria, Porphyromonas gingivalis (Yoshimura et al., 2000).

Methanethiol alongside with dimethyl sulfide are the main contributors of unpleasant odors that are developed by cruciferous vegetables such as broccolis, cabbage, radish and so on, which result in shorter shelf life to the food and decrease consumers acceptance (Engel et al., 2002).

Furthermore, dimethyl sulfide is released in significant amounts with other compounds as a volatile compound of paprika (Capsicum annuum) (Cremer and Eichner, 2000). It is also found in onion and garlic but it not important for the taste formation of these foods (Hattula and Granroth, 1974). 
Table 3. Chemical composition obtained from leaves in natura of Gallesia integrifolia by dynamic headspace.

\begin{tabular}{|c|c|c|c|}
\hline Peak & ${ }^{\mathrm{b}}$ Compounds & Area $(\%)$ & Identification method \\
\hline 1 & Dimethyl sulfide & 42.42 & $\mathrm{a}, \mathrm{b}$ \\
\hline 2 & 3-methylbutanal & 40.43 & $\mathrm{a}, \mathrm{b}$ \\
\hline 3 & Ethanol, 2-(methylthio) & 5.52 & $\mathrm{a}, \mathrm{b}$ \\
\hline 4 & Methyl disulfide & $\mathrm{t}$ & $\mathrm{a}, \mathrm{b}$ \\
\hline 5 & Dimethyl sulfone & $\mathrm{t}$ & $a, b$ \\
\hline 6 & 2-ethylfuran & $\mathrm{t}$ & $\mathrm{a}, \mathrm{b}$ \\
\hline 7 & 2,4-dithiapentane & $\mathrm{t}$ & $\mathrm{a}, \mathrm{b}$ \\
\hline 8 & Propanoic acid, 3-(methylthio) & $\mathrm{t}$ & $\mathrm{a}, \mathrm{b}$ \\
\hline 9 & 2,2-thiodiethanol & $\mathrm{t}$ & $\mathrm{a}, \mathrm{b}$ \\
\hline 10 & Methyl (methylsulfinyl) methyl sulfide (FAMSO) & $\mathrm{t}$ & $\mathrm{a}, \mathrm{b}$ \\
\hline 11 & Santene & $\mathrm{t}$ & $\mathrm{a}, \mathrm{b}$ \\
\hline 12 & Tricyclene & $\mathrm{t}$ & $\mathrm{a}, \mathrm{b}$ \\
\hline 13 & Artemesia triene & $\mathrm{t}$ & $\mathrm{a}, \mathrm{b}$ \\
\hline 14 & Thujene & $\mathrm{t}$ & $\mathrm{a}, \mathrm{b}$ \\
\hline 15 & $\alpha$-pinene & $\mathrm{t}$ & $a, b$ \\
\hline 16 & $\alpha$-fenchene & $\mathrm{t}$ & $a, b$ \\
\hline 17 & Camphene & $\mathrm{t}$ & $a, b$ \\
\hline 18 & Sabinene & $\mathrm{t}$ & $a, b$ \\
\hline 19 & $\beta$-pinene & $\mathrm{t}$ & $a, b$ \\
\hline 20 & Myrcene & $\mathrm{t}$ & $\mathrm{a}, \mathrm{b}$ \\
\hline 21 & 2-carene & $\mathrm{t}$ & $\mathrm{a}, \mathrm{b}$ \\
\hline 22 & $\alpha$-phellandrene & $\mathrm{t}$ & $\mathrm{a}, \mathrm{b}$ \\
\hline 23 & 3 -carene & $\mathrm{t}$ & $\mathrm{a}, \mathrm{b}$ \\
\hline 24 & Terpinene & $\mathrm{t}$ & $\mathrm{a}, \mathrm{b}$ \\
\hline 25 & Limonene & $\mathrm{t}$ & $\mathrm{a}, \mathrm{b}$ \\
\hline 26 & $\beta$-phellandrene & $\mathrm{t}$ & $\mathrm{a}, \mathrm{b}$ \\
\hline 27 & cis-ocimene & $\mathrm{t}$ & $\mathrm{a}, \mathrm{b}$ \\
\hline 28 & trans-ocimene & $\mathrm{t}$ & $\mathrm{a}, \mathrm{b}$ \\
\hline 29 & $\gamma$-terpinene & $\mathrm{t}$ & $\mathrm{a}, \mathrm{b}$ \\
\hline 30 & $\alpha$-terpinolene & 8.63 & $\mathrm{a}, \mathrm{b}$ \\
\hline 31 & Sabinene hydrate & $\mathrm{t}$ & $\mathrm{a}, \mathrm{b}$ \\
\hline 32 & Linalool & $\mathrm{t}$ & $\mathrm{a}, \mathrm{b}$ \\
\hline 33 & L-methionine & $\mathrm{t}$ & $\mathrm{a}, \mathrm{b}$ \\
\hline 34 & 2,7-dithiaoctane & $\mathrm{t}$ & $\mathrm{a}, \mathrm{b}$ \\
\hline 35 & Trithiometoxymethane & $\mathrm{t}$ & $\mathrm{a}, \mathrm{b}$ \\
\hline 36 & Myrtenol & $\mathrm{t}$ & $\mathrm{a}, \mathrm{b}$ \\
\hline 37 & Damascenone & $\mathrm{t}$ & $\mathrm{a}, \mathrm{b}$ \\
\hline 38 & trans-farnesene & $\mathrm{t}$ & $\mathrm{a}, \mathrm{b}$ \\
\hline 39 & cis-farnesol & $\mathrm{t}$ & $\mathrm{a}, \mathrm{b}$ \\
\hline 40 & trans-farnesol & $\mathrm{t}$ & $\mathrm{a}, \mathrm{b}$ \\
\hline 41 & 3,5-dithiahexanol-5,5-dioxide & $\mathrm{t}$ & $\mathrm{a}, \mathrm{b}$ \\
\hline 42 & Ethanol, 2-(octylthio) & $\mathrm{t}$ & $\mathrm{a}, \mathrm{b}$ \\
\hline 43 & 1,3-dimethyl-4-azaphenanthrene & $\mathrm{t}$ & $\mathrm{a}, \mathrm{b}$ \\
\hline 44 & 5-methyl-2-phenylindole & $\mathrm{t}$ & $\mathrm{a}, \mathrm{b}$ \\
\hline
\end{tabular}

44 5-methyl-2-phenylindole $\quad t \quad$ a,b

${ }^{\mathrm{a}}$ MS identification based on comparison of mass spectra using Wiley 275 libraries. ${ }^{\mathrm{b}}$ Compounds listed in order of elution in column HP-5MS. $\mathrm{t}=$ traces. Area $(\%)$ : percentage $(\%)$ of the area occupied by compounds within the chromatogram.

Dimethyl sulfide is the most abundant sulfur compound in beer, superior to the taste threshold of 30-45 $\mu \mathrm{L}$. In levels less than $100.00 \mu \mathrm{L}$ it contributes to the flavor characteristic of lager beer. However, in great amounts provides an unpleasant flavor of corn (Hansen, 1999). In Porto wine, aged samples developed aromas related to the presence of dimethyl sulfide (Silva Ferreira et al., 2003).

It is possible to obtain dimethyl sulfone from dimethyl sulfide (Fig 1), a major compound in in natura garlic wood fruits (Table 2). The process of dimethyl sulfone formation is possible because sulfides are easily oxidized into sulfones (Vollhardt and Schore, 2013) (Fig 5). Dimethyl sulfone is a compound that presents a simple structure and has already been isolated as a natural product in Cladonia deformis Hoffm liquen (Brunn and Sörensen, 1954). Moreover, dimethyl sulfone was also found in EO and extracts from garlic wood leaves (Barbosa et al., 1997).
Two aliphatic polysulfide were found as major ones in in natura garlic wood fruits (Table 1), 2,3,5-trithiahexane and 2,3,5,6-tetrathiaheptane (Fig 1), which have already been identified in EO from garlic oil leaves (Fraga et al., 2006). There are studies that reported the presence of 2,3,5trithiahexane in cheeses, broccolis, cooked cabbage, cauliflower, Lentinus edodes Sing, Beaufort and Camembert and also in hop oil, which in latter was produced by the oxidation of another sulfur compound in the presence of methanethiol (Moir et al., 1990).

However, the EO of a plant has a great variety of compounds such as terpene hydro carbonates, aldehydes, amines among others (Lima et al., 2003; Simões et al., 2010). In in natura garlic wood leaves (Table 3 ), $\alpha$-terpinolene was found (Fig 2), which is a compound belong to the terpene hydrocarbonate class. The flavor and aroma of fruits are related (Campbell-Platt, 2015) to this compound. Also, 3methylbutanal was found (Fig 2), an aldehyde that is also 
Table 4. Chemical composition obtained from flowers in natura of Gallesia integrifolia by dynamic headspace.

\begin{tabular}{|c|c|c|c|}
\hline Peak & ${ }^{\mathrm{b}}$ Compounds & Area $(\%)$ & Identification method \\
\hline 1 & Methanethiol & 44.91 & $\mathrm{a}, \mathrm{b}$ \\
\hline 2 & Dimethyl sulfide & 43.72 & $a, b$ \\
\hline 3 & 2-butanamine & 4.70 & $a, b$ \\
\hline 4 & 3-methylbutanal & $\mathrm{t}$ & $a, b$ \\
\hline 5 & Methyl disulfide & $\mathrm{t}$ & $a, b$ \\
\hline 6 & Propanenitrile, 3-(methylthio) & $\mathrm{t}$ & $a, b$ \\
\hline 7 & 2,4-dithiapentane & $\mathrm{t}$ & $a, b$ \\
\hline 8 & Santene & $\mathrm{t}$ & $a, b$ \\
\hline 9 & Tricyclene & $\mathrm{t}$ & $a, b$ \\
\hline 10 & Artemesia triene & $\mathrm{t}$ & $a, b$ \\
\hline 11 & Thujene & $\mathrm{t}$ & $a, b$ \\
\hline 12 & Dimethyl trisulfide & $\mathrm{t}$ & $a, b$ \\
\hline 13 & $\alpha$-pinene & $\mathrm{t}$ & $a, b$ \\
\hline 14 & $\alpha$-fenchene & $\mathrm{t}$ & $a, b$ \\
\hline 15 & Camphene & $\mathrm{t}$ & $a, b$ \\
\hline 16 & Sabinene & $\mathrm{t}$ & $a, b$ \\
\hline 17 & $\beta$-pinene & $\mathrm{t}$ & $a, b$ \\
\hline 18 & trans-isolimonene & $\mathrm{t}$ & $a, b$ \\
\hline 19 & Myrcene & $\mathrm{t}$ & $a, b$ \\
\hline 20 & 2-carene & $\mathrm{t}$ & $a, b$ \\
\hline 21 & 3-carene & $\mathrm{t}$ & $a, b$ \\
\hline 22 & Limonene & $\mathrm{t}$ & $a, b$ \\
\hline 23 & $\beta$-phellandrene & $\mathrm{t}$ & $a, b$ \\
\hline 24 & cis-ocimene & $\mathrm{t}$ & $a, b$ \\
\hline 25 & trans-ocimene & $\mathrm{t}$ & $a, b$ \\
\hline 26 & $\gamma$-terpinene & 2.25 & $a, b$ \\
\hline 27 & $\alpha$-terpinolene & $\mathrm{t}$ & $a, b$ \\
\hline 28 & Linalool & $\mathrm{t}$ & $a, b$ \\
\hline 29 & Sabinene hydrate & $\mathrm{t}$ & $a, b$ \\
\hline 30 & Myrtenol & $\mathrm{t}$ & $a, b$ \\
\hline 31 & Safranal & $\mathrm{t}$ & $a, b$ \\
\hline 32 & Sabinyl acetate & $\mathrm{t}$ & $a, b$ \\
\hline 33 & Neryl acetate & $\mathrm{t}$ & $a, b$ \\
\hline 34 & Elemene & $\mathrm{t}$ & $a, b$ \\
\hline 35 & $\beta$-caryophyllene & $\mathrm{t}$ & $a, b$ \\
\hline 36 & $\alpha$-humulene & $\mathrm{t}$ & $a, b$ \\
\hline 37 & 2,3,5-trithiahexane & $\mathrm{t}$ & $a, b$ \\
\hline 38 & Bisabolene & $\mathrm{t}$ & $a, b$ \\
\hline 39 & Caryophyllene oxide & $\mathrm{t}$ & $a, b$ \\
\hline 40 & $\beta$-sinensal & $\mathrm{t}$ & $a, b$ \\
\hline 41 & cis-trans-farnesol & $\mathrm{t}$ & $a, b$ \\
\hline 42 & $\alpha$-sinensal & $\mathrm{t}$ & $a, b$ \\
\hline 43 & N-ethyl-1,3-dithioisoindole & 3.69 & $a, b$ \\
\hline 44 & 3,4-dimetoxy-dl-phenylalamine & 0.73 & $a, b$ \\
\hline
\end{tabular}

${ }^{a}$ MS identification based on comparison of mass spectra using Wiley 275 libraries. ${ }^{b}$ Compounds listed in order of elution in column HP-5MS. $\mathrm{t}=$ traces. Area $(\%)$ : percentage $(\%)$ of the area occupied by compounds within the chromatogram.

Table 5. Averages \pm standard error of female mortality (\%), egg mass (g), hatchability (\%), reproductive efficiency (\%) and product efficiency (\%) in engorged Rhipicephalus (Boophilus) microplus females by Adult Immersion Test of essential oil (EO) of the fruits of Gallesia integrifolia.

\begin{tabular}{llllll}
$\begin{array}{l}\text { Concentration } \\
(\mathrm{mg} / \mathrm{mL})\end{array}$ & $\begin{array}{l}\text { Mortality } \\
\text { females }(\%)\end{array}$ & $\begin{array}{l}\text { ofMass of } \\
\text { females }(\mathrm{g})\end{array}$ & $\begin{array}{l}\text { Egg mass } \\
(\mathrm{g})\end{array}$ & $\begin{array}{l}\text { Hatchability } \\
(\%)\end{array}$ & $\begin{array}{l}\text { Product } \\
\text { efficiency }(\%)\end{array}$ \\
\hline PC & $100.00^{\mathrm{a}} \pm 0.00$ & $0.19^{\mathrm{a}} \pm 0.02$ & $0.00^{\mathrm{a}} \pm 0.00$ & $0.00^{\mathrm{a}} \pm 0.00$ & $100.00^{\mathrm{a}} \pm 0.00$ \\
300.00 & $100.00^{\mathrm{a}} \pm 0.00$ & $0.19^{\mathrm{a}} \pm 0.05$ & $0.00^{\mathrm{a}} \pm 0.00$ & $0.00^{\mathrm{a}} \pm 0.00$ & $100.00^{\mathrm{a}} \pm 0.00$ \\
200.00 & $96.67^{\mathrm{a}} \pm 3.33$ & $0.17^{\mathrm{a}} \pm 0.04$ & $0.0002^{\mathrm{a}} \pm 0.001$ & $3.35^{\mathrm{a}} \pm 0.35$ & $100.00^{\mathrm{a}} \pm 0.00$ \\
100.00 & $53.33^{\mathrm{b}} \pm 14.53$ & $0.19^{\mathrm{a}} \pm 0.04$ & $0.02^{\mathrm{b}} \pm 0.02$ & $29.55^{\mathrm{b}} \pm 7.20$ & $93.97^{\mathrm{a}} \pm 3.34$ \\
60.00 & $43.33^{\mathrm{c}} \pm 8.82$ & $0.19^{\mathrm{a}} \pm 0.02$ & $0.03^{\mathrm{b}} \pm 0.02$ & $46.11^{\mathrm{b}} \pm 18.85$ & $81.92^{\mathrm{b}} \pm 9.27$ \\
20.00 & $16.67^{\mathrm{d}} \pm 6.67$ & $0.19^{\mathrm{a}} \pm 0.03$ & $0.05^{\mathrm{c}} \pm 0.02$ & $74.77^{\mathrm{c}} \pm 8.24$ & $64.30^{\mathrm{b}} \pm 6.50$ \\
10.00 & $13.33^{\mathrm{d}} \pm 3.33$ & $0.18^{\mathrm{a}} \pm 0.03$ & $0.06^{\mathrm{c}} \pm 0.02$ & $77.06^{\mathrm{c}} \pm 3.37$ & $47.92^{\mathrm{c}} \pm 6.63$ \\
5.00 & $13.33^{\mathrm{d}} \pm 6.67$ & $0.18^{\mathrm{a}} \pm 0.02$ & $0.07^{\mathrm{c}} \pm 0.02$ & $76.63^{\mathrm{c}} \pm 6.52$ & $40.80^{\mathrm{c}} \pm 9.50$ \\
2.50 & $10.00^{\mathrm{d}} \pm 5.77$ & $0.14^{\mathrm{a}} \pm 0.04$ & $0.05^{\mathrm{c}} \pm 0.03$ & $78.48^{\mathrm{c}} \pm 6.41$ & $39.05^{\mathrm{c}} \pm 13.24$ \\
1.25 & $10.00^{\mathrm{d}} \pm 5.77$ & $0.17^{\mathrm{a}} \pm 0.03$ & $0.08^{\mathrm{d}} \pm 0.02$ & $81.40^{\mathrm{c}} \pm 6.10$ & $26.15^{\mathrm{d}} \pm 9.06$ \\
0.62 & $10.00^{\mathrm{d}} \pm 0.00$ & $0.15^{\mathrm{a}} \pm 0.03$ & $0.08^{\mathrm{d}} \pm 0.02$ & $83.50^{\mathrm{c}} \pm 3.86$ & $18.43^{\mathrm{d}} \pm 1.75$ \\
0.31 & $10.00^{\mathrm{d}} \pm 0.00$ & $0.17^{\mathrm{a}} \pm 0.03$ & $0.07^{\mathrm{d}} \pm 0.02$ & $85.64^{\mathrm{c}} \pm 2.52$ & $26.06^{\mathrm{d}} \pm 6.04$ \\
0.16 & $6.67^{\mathrm{d}} \pm 3.33$ & $0.16^{\mathrm{a}} \pm 0.02$ & $0.07^{\mathrm{d}} \pm 0.02$ & $85.91^{\mathrm{c}} \pm 3.46$ & $25.07^{\mathrm{d}} \pm 1.53$ \\
0.08 & $0.00^{\mathrm{d}} \pm 0.00$ & $0.15^{\mathrm{a}} \pm 0.02$ & $0.07^{\mathrm{d}} \pm 0.02$ & $88.58^{\mathrm{c}} \pm 3.68$ & $18.32^{\mathrm{d}} \pm 4.21$ \\
0.04 & $0.00^{\mathrm{d}} \pm 0.00$ & $0.16^{\mathrm{a}} \pm 0.03$ & $0.09^{\mathrm{d}} \pm 0.02$ & $89.02^{\mathrm{c}} \pm 4.67$ & $9.89^{\mathrm{d}} \pm 3.67$ \\
0.02 & $0.00^{\mathrm{d}} \pm 0.00$ & $0.17^{\mathrm{a}} \pm 0.02$ & $0.09^{\mathrm{d}} \pm 0.01$ & $93.99^{\mathrm{c}} \pm 1.32$ & $4.28^{\mathrm{d}} \pm 1.66$ \\
$N C$ & $0.00^{\mathrm{d}} \pm 0.00$ & $0.20^{\mathrm{a}} \pm 0.02$ & $0.09^{\mathrm{d}} \pm 0.01$ & $100.00^{\mathrm{c}} \pm 0.00$ & $0.00^{\mathrm{d}} \pm 0.00$ \\
\hline
\end{tabular}

PC = positive control ( $0.125 \%$ commercial solution containing $15.00 \%$ cypermethrin, $25.00 \%$ chlorpyrifos and $1.00 \%$ citronellal); NC = negative control (polysorbate $(80)$ at $2.00 \%$ solution). Means followed by the same letter do not differ from each other by the Scott-Knott test p (0.05). 
Table 6. Averages \pm standard error of female mortality (\%), egg mass (g), hatchability (\%), reproductive efficiency $(\%)$ and product efficiency (\%) in engorged Rhipicephalus (Boophilus) microplus females by Adult Immersion Test of essential oil (EO) of the leaves of Gallesia integrifolia.

\begin{tabular}{|c|c|c|c|c|c|}
\hline $\begin{array}{l}\text { Concentration } \\
(\mathrm{mg} / \mathrm{mL})\end{array}$ & $\begin{array}{l}\text { Mortality of } \\
\text { females (\%) }\end{array}$ & $\begin{array}{c}\text { Mass of } \\
\text { females }(\mathrm{g})\end{array}$ & $\begin{array}{l}\text { Egg mass } \\
(\mathrm{g})\end{array}$ & $\begin{array}{c}\text { Hatchability } \\
(\%)\end{array}$ & $\begin{array}{c}\text { Product } \\
\text { efficiency }(\%)\end{array}$ \\
\hline $\mathrm{PC}$ & $100.00^{\mathrm{a}} \pm 0.00$ & $0.19^{\mathrm{a}} \pm 0.02$ & $0.00^{\mathrm{a}} \pm 0.00$ & $0.00^{\mathrm{a}} \pm 0.00$ & $100.00^{\mathrm{a}} \pm 0.00$ \\
\hline 500.00 & $100.00^{\mathrm{a}} \pm 0.00$ & $0.17^{a} \pm 0.02$ & $0.00^{\mathrm{a}} \pm 0.00$ & $0.00^{\mathrm{a}} \pm 0.00$ & $100.00^{\mathrm{a}} \pm 0.00$ \\
\hline 400.00 & $100.00^{\mathrm{a}} \pm 0.00$ & $0.17^{\mathrm{a}} \pm 0.02$ & $0.00^{\mathrm{a}} \pm 0.00$ & $0.00^{\mathrm{a}} \pm 0.00$ & $100.00^{\mathrm{a}} \pm 0.00$ \\
\hline 300.00 & $93.33^{\mathrm{a}} \pm 3.33$ & $0.17^{\mathrm{a}} \pm 0.02$ & $0.00^{\mathrm{a}} \pm 0.00$ & $0.00^{\mathrm{a}} \pm 0.00$ & $100.00^{\mathrm{a}} \pm 0.00$ \\
\hline 200.00 & $86.67^{\mathrm{a}} \pm 8.82$ & $0.16^{\mathrm{a}} \pm 0.03$ & $0.01^{\mathrm{a}} \pm 0.01$ & $5.49^{\mathrm{a}} \pm 0.00$ & $100.00^{\mathrm{a}} \pm 0.30$ \\
\hline 100.00 & $63.33^{\mathrm{b}} \pm 6.67$ & $0.16^{\mathrm{a}} \pm 0.03$ & $0.03^{\mathrm{b}} \pm 0.02$ & $18.86^{\mathrm{b}} \pm 2.65$ & $93.97^{\mathrm{a}} \pm 2.00$ \\
\hline 60.00 & $40.00^{c} \pm 20.00$ & $0.16^{\mathrm{a}} \pm 0.03$ & $0.01^{\mathrm{a}} \pm 0.01$ & $24.82^{\mathrm{b}} \pm 6.34$ & $81.92^{\mathrm{b}} \pm 1.93$ \\
\hline 20.00 & $36.67^{\mathrm{c}} \pm 6.67$ & $0.16^{\mathrm{a}} \pm 0.02$ & $0.03^{\mathrm{b}} \pm 0.02$ & $31.23^{\mathrm{b}} \pm 4.60$ & $64.30^{\mathrm{b}} \pm 2.74$ \\
\hline 10.00 & $30.00^{c} \pm 0.00$ & $0.16^{\mathrm{a}} \pm 0.02$ & $0.05^{\mathrm{c}} \pm 0.01$ & $43.01^{c} \pm 7.63$ & $47.92^{\mathrm{c}} \pm 5.82$ \\
\hline 5.00 & $26.67^{\mathrm{c}} \pm 17.64$ & $0.16^{\mathrm{a}} \pm 0.03$ & $0.05^{\mathrm{c}} \pm 0.02$ & $50.73^{\mathrm{d}} \pm 1.11$ & $40.80^{\mathrm{c}} \pm 3.87$ \\
\hline 2.50 & $13.33^{\mathrm{d}} \pm 13.33$ & $0.16^{\mathrm{a}} \pm 0.02$ & $0.04^{c} \pm 0.01$ & $81.28^{\mathrm{e}} \pm 6.44$ & $39.05^{\mathrm{c}} \pm 3.65$ \\
\hline 1.25 & $10.00^{\mathrm{d}} \pm 0.00$ & $0.16^{\mathrm{a}} \pm 0.03$ & $0.04^{\mathrm{c}} \pm 0.02$ & $83.30^{\mathrm{e}} \pm 7.57$ & $26.15^{\mathrm{d}} \pm 4.52$ \\
\hline 0.62 & $10.00^{\mathrm{d}} \pm 10.00$ & $0.17^{\mathrm{a}} \pm 0.02$ & $0.04^{c} \pm 0.02$ & $82.16^{\mathrm{e}} \pm 4.28$ & $18.43^{\mathrm{d}} \pm 7.61$ \\
\hline 0.31 & $10.00^{\mathrm{d}} \pm 0.00$ & $0.17^{\mathrm{a}} \pm 0.03$ & $0.05^{\mathrm{c}} \pm 0.02$ & $84.42^{\mathrm{e}} \pm 3.16$ & $26.06^{\mathrm{d}} \pm 4.58$ \\
\hline 0.16 & $10.00^{\mathrm{d}} \pm 5.77$ & $0.17^{\mathrm{a}} \pm 0.02$ & $0.07^{\mathrm{c}} \pm 0.02$ & $84.90^{\mathrm{e}} \pm 3.96$ & $25.07^{\mathrm{d}} \pm 4.62$ \\
\hline 0.08 & $6.67^{\mathrm{d}} \pm 3.33$ & $0.17^{\mathrm{a}} \pm 0.03$ & $0.08^{\mathrm{d}} \pm 0.02$ & $84.48^{\mathrm{e}} \pm 0.96$ & $18.32^{\mathrm{d}} \pm 3.58$ \\
\hline 0.04 & $6.67^{\mathrm{d}} \pm 3.33$ & $0.16^{\mathrm{a}} \pm 0.02$ & $0.08^{\mathrm{d}} \pm 0.02$ & $85.46^{\mathrm{e}} \pm 4.81$ & $9.89^{\mathrm{d}} \pm 9.74$ \\
\hline 0.02 & $6.67^{\mathrm{d}} \pm 6.67$ & $0.16^{\mathrm{a}} \pm 0.02$ & $0.08^{\mathrm{d}} \pm 0.02$ & $86.84^{\mathrm{e}} \pm 3.84$ & $4.28^{\mathrm{d}} \pm 9.83$ \\
\hline $\mathrm{NC}$ & $0.00^{\mathrm{d}} \pm 0.00$ & $0.20^{\mathrm{a}} \pm 0.02$ & $0.09^{\mathrm{d}} \pm 0.01$ & $100.00^{\mathrm{e}} \pm 0.00$ & $0.00^{\mathrm{d}} \pm 0.00$ \\
\hline
\end{tabular}

$\mathrm{PC}=$ positive control ( $0.125 \%$ commercial solution containing $15.00 \%$ cypermethrin, $25.00 \%$ chlorpyrifos and $1.00 \%$ citronellal); NC = negative control (polysorbate $(80)$ at $2.00 \%$ solution). Means followed by the same letter do not differ from each other by the Scott-Knott test p (0.05).

Table 7. Averages \pm standard error of female mortality (\%), egg mass (g), hatchability (\%), reproductive efficiency (\%) and product efficiency (\%) in engorged Rhipicephalus (Boophilus) microplus females by Adult Immersion Test of essential oil (EO) of the flowers of Gallesia integrifolia.

\begin{tabular}{|c|c|c|c|c|c|}
\hline $\begin{array}{l}\text { Concentration } \\
(\mathrm{mg} / \mathrm{mL})\end{array}$ & $\begin{array}{l}\text { Mortality of } \\
\text { females (\%) }\end{array}$ & $\begin{array}{c}\text { Mass of } \\
\text { females }(\mathrm{g})\end{array}$ & $\begin{array}{c}\text { Egg mass } \\
(\mathrm{g})\end{array}$ & $\begin{array}{c}\text { Hatchability } \\
(\%)\end{array}$ & $\begin{array}{c}\text { Product } \\
\text { efficiency }(\%)\end{array}$ \\
\hline $\mathrm{PC}$ & $100.00^{\mathrm{a}} \pm 0.00$ & $0.19^{\mathrm{a}} \pm 0.02$ & $0.00^{\mathrm{a}} \pm 0.00$ & $0.00^{\mathrm{a}} \pm 0.00$ & $100.00^{\mathrm{a}} \pm 0.00$ \\
\hline 500.00 & $100.00^{\mathrm{a}} \pm 0.00$ & $0.18^{a} \pm 0.02$ & $0.00^{\mathrm{a}} \pm 0.00$ & $0.00^{\mathrm{a}} \pm 0.00$ & $100.00^{a} \pm 0.00$ \\
\hline 400.00 & $100.00^{\mathrm{a}} \pm 0.00$ & $0.20^{\mathrm{a}} \pm 0.02$ & $0.00^{\mathrm{a}} \pm 0.00$ & $0.00^{\mathrm{a}} \pm 0.00$ & $100.00^{a} \pm 0.00$ \\
\hline 300.00 & $100.00^{\mathrm{a}} \pm 0.00$ & $0.19^{\mathrm{a}} \pm 0.02$ & $0.00^{\mathrm{a}} \pm 0.00$ & $0.00^{\mathrm{a}} \pm 0.00$ & $100.00^{\mathrm{a}} \pm 0.00$ \\
\hline 200.00 & $93.33^{\mathrm{a}} \pm 1.05$ & $0.20^{\mathrm{a}} \pm 0.02$ & $0.00^{\mathrm{a}} \pm 0.00$ & $0.00^{\mathrm{a}} \pm 0.00$ & $100.00^{\mathrm{a}} \pm 0.00$ \\
\hline 100.00 & $76.67^{b} \pm 2.79$ & $0.19^{a} \pm 0.03$ & $0.01^{\mathrm{a}} \pm 0.01$ & $8.33^{\mathrm{a}} \pm 6.90$ & $98.67^{\mathrm{a}} \pm 1.21$ \\
\hline 60.00 & $26.67^{c} \pm 2.79$ & $0.20^{\mathrm{a}} \pm 0.02$ & $0.04^{\mathrm{b}} \pm 0.01$ & $63.66^{\mathrm{b}} \pm 8.24$ & $72.74^{b} \pm 2.20$ \\
\hline 20.00 & $20.00^{c} \pm 0.00$ & $0.19^{\mathrm{a}} \pm 0.02$ & $0.05^{\mathrm{b}} \pm 0.01$ & $68.69^{\mathrm{b}} \pm 1.41$ & $61.95^{\mathrm{c}} \pm 3.40$ \\
\hline 10.00 & $20.00^{c} \pm 3.16$ & $0.20^{\mathrm{a}} \pm 0.02$ & $0.06^{\mathrm{c}} \pm 0.02$ & $67.79^{b} \pm 8.75$ & $58.38^{c} \pm 8.12$ \\
\hline 5.00 & $20.00^{c} \pm 1.83$ & $0.20^{\mathrm{a}} \pm 0.02$ & $0.07^{\mathrm{c}} \pm 0.02$ & $70.69^{b} \pm 2.74$ & $51.85^{\mathrm{c}} \pm 8.76$ \\
\hline 2.50 & $13.33^{c} \pm 2.79$ & $0.20^{\mathrm{a}} \pm 0.02$ & $0.07^{\mathrm{c}} \pm 0.02$ & $70.60^{b} \pm 7.03$ & $49.82^{c} \pm 9.80$ \\
\hline 1.25 & $13.33^{\mathrm{c}} \pm 1.05$ & $0.19^{\mathrm{a}} \pm 0.02$ & $0.07^{\mathrm{c}} \pm 0.02$ & $72.71^{b} \pm 3.96$ & $48.23^{c} \pm 3.74$ \\
\hline 0.62 & $13.33^{c} \pm 2.11$ & $0.20^{\mathrm{a}} \pm 0.03$ & $0.08^{\mathrm{d}} \pm 0.02$ & $77.49^{b} \pm 3.51$ & $41.16^{\mathrm{c}} \pm 6.00$ \\
\hline 0.31 & $10.00^{\mathrm{c}} \pm 1.83$ & $0.20^{\mathrm{a}} \pm 0.02$ & $0.08^{\mathrm{d}} \pm 0.02$ & $79.63^{c} \pm 3.29$ & $35.45^{\mathrm{d}} \pm 5.18$ \\
\hline 0.16 & $10.00^{c} \pm 3.16$ & $0.20^{\mathrm{a}} \pm 0.02$ & $0.10^{\mathrm{d}} \pm 0.02$ & $81.76^{\mathrm{c}} \pm 1.35$ & $23.08^{\mathrm{d}} \pm 3.35$ \\
\hline 0.08 & $6.67^{c} \pm 2.11$ & $0.19^{\mathrm{a}} \pm 0.02$ & $0.08^{\mathrm{d}} \pm 0.02$ & $88.26^{\mathrm{c}} \pm 4.91$ & $26.91^{\mathrm{d}} \pm 11.06$ \\
\hline 0.04 & $6.67^{\mathrm{c}} \pm 1.05$ & $0.18^{\mathrm{a}} \pm 0.02$ & $0.08^{\mathrm{d}} \pm 0.02$ & $92.34^{c} \pm 7.15$ & $22.86^{\mathrm{d}} \pm 5.53$ \\
\hline 0.02 & $6.67^{\mathrm{c}} \pm 1.05$ & $0.19^{\mathrm{a}} \pm 0.02$ & $0.09^{\mathrm{d}} \pm 0.02$ & $92.25^{\mathrm{c}} \pm 0.16$ & $20.94^{\mathrm{d}} \pm 8.47$ \\
\hline $\mathrm{NC}$ & $0.00^{\mathrm{c}} \pm 0.00$ & $0.20^{\mathrm{a}} \pm 0.02$ & $0.09^{\mathrm{d}} \pm 0.02$ & $100.00^{c} \pm 0.00$ & $0.00^{\mathrm{d}} \pm 0.00$ \\
\hline
\end{tabular}

$\mathrm{PC}=$ positive control $(0.125 \%$ commercial solution containing $15.00 \%$ cypermethrin, $25.00 \%$ chlorpyrifos and $1.00 \%$ citronellal); NC $=$ negative control (polysorbate $(80)$ at $2.00 \%$ solution). Means followed by the same letter do not differ from each other by the Scott-Knott test $\mathrm{p}(0.05)$.

Table 8. Averages \pm standard error of Lethal Doses $\mathrm{LD}_{50}$ and $\mathrm{LD} 99.9(\mathrm{mg} / \mathrm{mL})$ and confidence interval (CI) of essential oil (EO) of fruits, leaves and flowers of Gallesia integrifolia on larvae and adults of the Rhipicephalus (Boophilus) microplus by Probitos analysis.

\begin{tabular}{|c|c|c|c|c|c|c|}
\hline & \multicolumn{2}{|c|}{ EO of FRUITS } & \multicolumn{2}{|c|}{ EO of LEAVES } & \multicolumn{2}{|c|}{ EO of FLOWERS } \\
\hline & $\begin{array}{l}\mathrm{LD}_{50} \\
\text { (CI) }\end{array}$ & $\begin{array}{c}\mathrm{LD}_{99.9} \\
(\mathrm{CI})\end{array}$ & $\begin{array}{l}\mathrm{LD}_{50} \\
(\mathrm{CI})\end{array}$ & $\begin{array}{c}\mathrm{LD}_{99.9} \\
(\mathrm{CI})\end{array}$ & $\begin{array}{l}\mathrm{LD}_{50} \\
(\mathrm{CI})\end{array}$ & $\begin{array}{c}\mathrm{LD}_{99.9} \\
(\mathrm{CI})\end{array}$ \\
\hline $\begin{array}{l}\text { Mortality of } \\
\text { Larvae } \\
\text { Mortality of } \\
\text { Females }\end{array}$ & $\begin{array}{c}0.06^{\mathrm{a}} \pm 0.00 \\
(0.06-0.07) \\
114.82^{\mathrm{a}} \pm 5.00 \\
(104.91-120.88)\end{array}$ & $\begin{array}{c}0.23^{\mathrm{A}} \pm 0.01 \\
(0.22-0.24) \\
251.59^{\mathrm{A}} \pm 5.14 \\
(243.00-260.76)\end{array}$ & $\begin{array}{c}0.99^{\mathrm{b}} \pm 0.12 \\
(0.75-1.12) \\
150.55^{\mathrm{b}} \pm 4.17 \\
(144.29-158.45)\end{array}$ & $\begin{array}{c}2.15^{\mathrm{B}} \pm 0.11 \\
(1.95-2.31) \\
385.68^{\mathrm{B}} \pm 4.65 \\
(376.49-391.44)\end{array}$ & $\begin{array}{c}0.05^{\mathrm{a}} \pm 0.00 \\
(0.04-0.05) \\
150.97^{\mathrm{b}} \pm 4.77 \\
(141.45-156.08)\end{array}$ & $\begin{array}{c}0.08^{\mathrm{A}} \pm 0.00 \\
(0.08-0.09) \\
373.92^{\mathrm{B}} \pm 1.77 \\
(371.15-377.21)\end{array}$ \\
\hline
\end{tabular}

$\mathrm{LD}_{50}$ : Lethal Dose 50\%; $\mathrm{LD}_{99.9}$ Lethal Dose $99.9 \%$; CI: Confidence interval. Means followed by the same letter in the lines do not differ from each other by the Scott-Knott test $\mathrm{p}(0.05)$. 
present in cauliflower (Van Langenhove et al., 1991). Furthermore, 2-butanamine was found (Fig 3) as one of the major compounds of in natura garlic word flowers (Table 4). Plants produce a lot of important amine compounds but they are known to have very unpleasant odors (Winter, 2011).

\section{Acaricidal activity on Rhipichephalus (Boophilus) microplus}

There are no studies reporting the acaricidal properties for the application of garlic wood EO from fruits, leaves and flowers. However, according to Reis et al. (2015), sulfur compounds can have acaricidal and fungicidal effects, which corroborate studies done on Petiveria alliacea, a plant from the same family as garlic wood and that also has sulfur compounds in its chemical composition such as dibenzyl trisulfide, which separately presented high acaricidal potential (Lyndon et al., 1997; Rosado-Aguillar et al., 2010; Kerudo et al., 2015). This effect was also confirmed in another study on $R$. (B.) microplus utilizing raw extract from $P$. alliacea stems at the concentration of $200.00 \mathrm{mg} / \mathrm{mL}$, which showed a mortality rate of $(86.60 \pm 15.20 \%)$ on female ticks. Regarding the egg hatching inhibition, the raw extract from $P$. alliacea leaves provided an inhibition of $(26.00 \pm$ $5.20 \%$ ) at the concentration of $100 \mathrm{mg} / \mathrm{mL}$ (Rosado-Aguillar et al, 2010).

Compared to the present study, the EO from garlic wood fruits presented a mortality rate of $(96.67 \pm 3.33 \%)$ on female ticks at the concentration of $200.00 \mathrm{mg} / \mathrm{mL}$ (Table 5) while the EO from leaves at the concentration of $100 \mathrm{mg} / \mathrm{mL}$ presented an inhibition of egg hatching of $81.14 \pm 2.65 \%$ (Table 6), making evident that garlic wood EO was more active compared to $P$. alliacea.

Table 9 shows that EO of garlic wood provided greater activity on $R$. (B.) microplus larvae. This may have happened due to the fact that larvae presented greater vulnerability than adult. In this case, the EO could act by inhibiting chitin, the greatest compound in tick cuticle, which would prevent stage change, growth and larva hatching (Santos et al., 2015). The pest might also be killed by exhaustion, an effect caused by EO viscosity, and by asphyxiation when EO overlaps the larva body surface (Prates et al., 1993). On the other hand, in the adult stage, the EO action is more difficult because in this cycle stage the chitin layer is already completely formed, making the penetration of EO difficult.

Comparison of garlic wood EO from fruits, leaves and flowers shows that EO from fruits causes a better mortality rate on female ticks (Table 8), whereas the inhibition of egg hatching was observed utilizing EO of garlic wood leaves (Table 6).

\section{Larvicidal activity on Rhipichephalus (Boophilus) microplus}

Comparison of wood EO extracted from fruits leaves and flowers verified that $\mathrm{EO}$ from flowers can have better control since all larvae $(100.00 \%)$ were killed at a concentration of $0.08 \mathrm{mg} / \mathrm{mL}$ (Table 8 ). For the larvicidal activity on $R$. (B) microplus there have not yet been studies to report this property of garlic wood EO from fruits, leaves and flowers. However, this activity was also done with raw extracts of $P$. alliacea (Phytolaccaceae) leaves and stems.

The larvicidal activity done with the raw extract of $P$. alliacea leaves showed ( $\mathrm{LD}_{99.9}$ of $\left.12.29 \mathrm{mg} / \mathrm{mL}\right)$, while for the raw extract from stem ( $\mathrm{LD}_{99.9}$ of $16.52 \mathrm{mg} / \mathrm{mL}$ ) (RosadoAguillar et al., 2010) was obtained. The results obtained by Rosado-Aguillar and collaborators (2010), compared the garlic wood EO from fruits, leaves and flowers (Table 8). It is verified that garlic wood EO (fruits, leaves and flowers) provided better results and consequently a better larvicidal activity.

\section{Materials and Methods}

\section{Plant material}

Garlic wood fruits, leaves and flowers were harvested in the city of Umuarama - PR, at the coordinates S23 $46^{\prime} 16^{\prime \prime}$ and WO53 ${ }^{\circ} 19^{\prime} 38^{\prime \prime}$, and $442 \mathrm{~m}$ of altitude. Fruits were harvested from May to June, 2015; leaves and flowers in December, 2015.

\section{Extraction of garlic wood essential oil (EO)}

Fruits, leaves and fresh flowers of garlic wood were separately utilized to extract the essential oil by hydrodistillation using a Clevenger-type apparatus for $2 \mathrm{~h}$. The oil was removed from the equipment with PA hexane using a Pasteur pipette and dried with anhydrous sodium sulfate $\left(\mathrm{Na}_{2} \mathrm{SO}_{4}\right)$ (Simões et al., 2010), and then stored in amber flasks which were kept under refrigeration at $\left(-4{ }^{\circ} \mathrm{C}\right)$ (Omolo et al., 2004).

\section{Physical and chemical indices of garlic wood essential oil}

\section{Relative density}

The relative density of a substance is the ratio of its mass by its volume at $20{ }^{\circ} \mathrm{C}$. It is determined according to the technique described in the Brazilian Pharmacopeia (Farmacopeia, 1988).

\section{Refraction index}

The refraction index was determined by an $\mathrm{ABBE}$ refractometer, model RL3, PZO Warszawa, at $20{ }^{\circ} \mathrm{C}$ (Farmacopeia, 1988).

\section{Chemical characterization of garlic wood essential oil}

Five grams of in natura garlic wood vegetal material (fruits, leaves and flowers) were stored in vials for dynamic headspace. The parameters for headspace performance were: incubation temperature $\left(100{ }^{\circ} \mathrm{C}\right)$, incubation time $(30 \mathrm{~min})$, agitation (on for $1 \mathrm{~min}$; off for $10 \mathrm{~s}$ ), syringe temperature $\left(150{ }^{\circ} \mathrm{C}\right)$ and agitation speed $(500 \mathrm{rpm})$. The volatile compounds were analyzed by gas chromatography/mass spectrometry - GC/MS, using an Agilent chromatographer, model 19091S-433, coupled to an Agilent mass spectrometer, model 19091J-433. An HP-5MS 5\% (30 m x $0.25 \mathrm{~mm}$ x 0.25 $\mu \mathrm{m})$ analytical column was utilized with initial temperature of $60{ }^{\circ} \mathrm{C}$ and kept for $3 \mathrm{~min}$. Then, the temperature raised to $300{ }^{\circ} \mathrm{C}$ with a ramp of $5{ }^{\circ} \mathrm{C} / \mathrm{min}$ and remained in that temperature for $10 \mathrm{~min}$, and finally reached $310{ }^{\circ} \mathrm{C}$ with a ramp of $10{ }^{\circ} \mathrm{C} / \mathrm{min}$ for $10 \mathrm{~min}$. The utilized carrier gas was helium with a linear velocity of $1 \mathrm{~mL} / \mathrm{min}$ until $300{ }^{\circ} \mathrm{C}$, and pressure release of $56 \mathrm{kPa}$. The injector temperature was 300 ${ }^{\circ} \mathrm{C}$. The injection volume was $2 \mu \mathrm{L}$. The injection was done in split mode (20:1). The transfer line was kept at $285^{\circ} \mathrm{C}$ and the ionization and quadrupole sources were at $230{ }^{\circ} \mathrm{C}$ and $150{ }^{\circ} \mathrm{C}$, respectively. The detection system was EM in "Scan" mode, in the mass/charge ratio $(\mathrm{m} / \mathrm{z})$ of $40-550$ band, with "Solvent Delay" of $3 \mathrm{~min}$. The compounds found in in natura vegetal material (fruits, leaves and flowers) of garlic 
wood were identified by comparing its mass spectra with the mass spectra from WILEY 275 libraries (Adams, 2012).

\section{Acaricidal and larvicidal activity of garlic wood essential oil on Rhipicephalus (Boophilus) microplus}

The acaricidal activity of garlic wood essential oil from fruits, leaves and flowers were determined by Adult Immersion Test and Larval Packet Test as recommended by the Drummond et al. (1973) and Leite et al. (1995).

\section{Harvesting of ingurgitated females}

$1550 R$. (B.) microplus female ticks were randomly harvested from milk cattle with no spraying treatment against ticks for over 60 days, belonged to the Veterinary Hospital of Paranaense Univesity - UNIPAR, Umuarama - PR. The ticks were transported in a container with appropriate air circulation in the laboratory of Natural Products of UNIPAR.

\section{Treatment groups}

The ticks were selected considering the following aspects: normal appearance and motility, wholesome body and maximum ingurgitation. Then, they were cleaned with purified water (Leite et al., 1995) and were divided in groups of ten. Sixteen dilutions of EO from fruits, leaves and flowers were prepared in concentrations that varied from 500.00 to $0.02 \mathrm{mg} / \mathrm{mL}$, utilizing polysorbate (80) at $2.00 \%(\mathrm{v} / \mathrm{v})$ as emulsifier and purified water as solvent. As negative control, purified water and polysorbate (80) at $2.00 \%(\mathrm{v} / \mathrm{v})$ were used, and as positive control, a commercial solution at $0.12 \%$ containing $15.00 \%$ of cipermetrine, $25.00 \%$ of chlorpyrifos and $1.00 \%$ of citronellal was utilized. The treatments were done in triplicate.

\section{Sensitivity of ingurgitated females in adult immersion test (AIT)}

According to the methodology described by Drummond and collaborators (1973), groups of 10 female ticks were weighed and immersed for 5 minutes in each treatment. They were dried in paper and distributed in a Petri dish, then were identified and labelled. The Petri dishes were stored in a styrofoam box containing moist cotton and kept for 14 days for egg laying.

After 14 days, the mass of eggs from each female tick was weighed and transferred to assay tubes appropriately (each female and its eggs). Tubes were placed in the styrofoam box containing moist cotton for 21 days to hatch.

After 21 days, the larvae were killed with sulfuric ether and counted using an entomologic loupe to verify the egg hatching rate. From data of female ticks' mass, egg mass and hatching percentage, the reproductive efficiency (RE) and product efficiency (PE) were determined by equations 1 and 2, respectively (Drummond et al., 1973).

$$
\begin{aligned}
& \mathrm{RE}=\frac{\text { Egg mass }(\mathrm{g}) \times \text { Hatching rate }(\%)}{\text { Female tick mass }} \times 20.000 \quad \text { Eq.1 }
\end{aligned}
$$

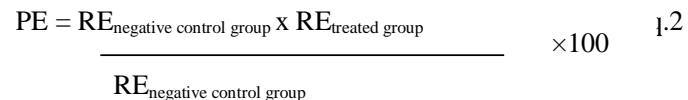

Larvicidal activity of garlic wood essential oil on
Rhipicephalus (Boophilus) microplus

The utilized technique was described by Leite and collaborators (Leite et al., 1995), in which 100 larvae were placed on $2.00 \times 2.00 \mathrm{~cm}$ paper filter that had freshly been moistened with dilutions of EO from fruits, leaves and flowers of garlic wood, forming a sealed "sandwich", stored in a Petri dish and kept at room temperature. The reading was done after $24 \mathrm{~h}$ when live and dead larvae were separated using an entomologic loupe.

Twenty dilutions of garlic wood EO from fruits, leaves and flowers were prepared in concentrations ranging from 10.00 to $0.0006 \mathrm{mg} / \mathrm{mL}$, utilizing polysorbate $(80)$ at $2.00 \%(\mathrm{v} / \mathrm{v})$ as emulsifier and purified water as solvent. As negative control, purified water and polysorbate (80) at $2.00 \%(\mathrm{v} / \mathrm{v})$ was utilized, whereas as positive control organophosphates from an acaricide (cipermetrine $15.00 \%$; chlorpyrifos $25.00 \%$; citronellal $1.00 \%$ ).

The treatments were done in triplicate and the larval mortality (LM) (Equation 3) and the average larval mortality were determined.

$\mathrm{LM}(\%)=\frac{\text { Dead larvae }}{\text { Total of larvae }} \times 100 \quad$ Eq.3

\section{Statistical analysis for the acaricidal and larvicidal activity}

The tests were done in triplicate and the mortality percentage (\%) of female ticks and larvae of $R$. (B.) microplus were obtained by calculating the average \pm standard error utilizing the Microsoft Excel ${ }^{\circledR}$ program (Excel® Version 2010). The experimental design was completely randomized (CRD). The data were submitted to analysis of variance (ANOVA) and compared utilizing Sisvar 5.6 program by Scott-Knott's test $(\mathrm{p}<0.05)$.

\section{Conclusion}

The essential oil (EO) from garlic wood fruit, leaves and flowers showed sulfur compounds in its chemical analysis, which were active in different phases of the cattle tick's reproductive cycle. EO from garlic wood in three periods (fruit, leaves and flowers) presented greater activity in the phase that corresponds to the larval cycle. However, when compared to the other phases of the cattle tick's cycles, the EO from fruits was more active on the females' mortality and the EO from leaves was more efficient on the inhibition of egg hatching. Thus, the garlic wood EO can be considered a promising agent to combat cattle tick, once it causes losses to livestock.

\section{Acknowledgements}

The authors thank CAPES, CNPq, Universidade Paranaense (UNIPAR), UNI-Cesumar and Instituto Federal do Paraná (IFPR)/Campus Umuarama for the support.

\section{References}

Adams RP (2012) Identification of essential oil componentes by Gas Chomatography/Mass Spectrometry, 4th edn. Allured Publishing Corporation. Illinois, USA.

Akisue MK, Akisue G, Oliveira FCC (1986) Pharmacognostic characterization of pau d'alho Gallesia integrifolia (Spreng.) Harms. Rev Bras Farmacogn. 1(2): 166-182. 
Álvarez V, Loaiza J, Bonilla R, Barrios M (2008) Control in vitro tick (Boophilus microplus; Acari: Ixodidae) through plant extracts. Rev Biol Trop. 56(1):291-302.

Barbosa LCA, Teixeira RR, Demuner AJ (1997) Vitamin E and other chemical constituents of the leaves of Gallesia gorazema. Fitoterapia. 68(6):514-519.

Block E, Putman D, Zhao SH (1992) Allium chemistry: GCMS analysis of thiosulfinates and related compounds from onion, leek, scallion, shallot, chive, and Chinese chive. J Agr Food Chem. 40(12):2431-2436.

Brasil Ministério da Agricultura. Portaria $\mathrm{n}^{\circ} 90$ de 04 de dezembro de 1989. Normas para produção, controle e utilização de produtos antiparasitários. Diário Oficial, 22 de janeiro de 1990, seção 1, coluna 2.

Brunn T, Sörensen NA (1954) A note on the occurrence of dimethyl sulphone in Cladonia deformis Hoffm. Acta Chem Scand. 8(4):703.

Burbank HM, Qian MC (2005) Volatiles sulfur compounds in Cheddar cheese determined by headspace solid-phase micro extraction and gas chromatograph-pulsed flame photometric detection. J Chromatogr A. 1066(1-2):149157.

Campbell-Platt G (2015) Ciência e tecnologia de alimentos, 1st edn. Manole. São Paulo, Brazil.

Cerri CC, Moreira CS, Alves PA, Raucci GS, Castigioni BA, Mello FFC, Cerri DGP, Cerri CEP (2015) Assessing the carbon foot print of beef cattle in Brazil: a case study with 22 farms in the State of Mato Grosso. J Clean Prod. 112(4):2593-2600.

Constantino MG, Da Silva GVJ, Donate PM (2004) Fundamentos de química experimental, 2nd edn. Universidade de São Paulo. São Paulo, Brazil.

Cremer DR, Eichner K (2000) Formation of volatile compounds during heating of spice paprika (Capsicum annuum) powder. J Agr Food Chem. 48(6):2454-2460.

De Souza LFG, Cecílio Filho AB, De Túlio FA, Nowaki RHD (2015) Effect of sulphur dose on the productivity and quality of onions. Aust J Crop Sci. 9(8):728-733.

Dewick PM (2002) Medicinal natural products: a biosynthetic approach, 2nd edn. John Wiley \& Sons Ltd. New York, USA.

Drummond RO, Ernest SE, Trevino JL, Gladney WJ, Grhham OH (1973) Boophilus annulatus and Boophilus microplus (Acarine: Ixodidae) in natural and experimental conditions. Folia Parasit. 37:331-336.

Durigan G, Figliolia MB, Kawabata M, Garrido MAO, Baitello JB (1997) Sementes e mudas de árvores tropicais, Páginas \& Letras. São Paulo, Brazil.

Engel E, Baty C, Lecorre D, Souchon I, Martin N (2002) Flavor-active compounds potentially implicated in cooked cauliflower acceptance. J Agr Food Chem. 50(22):64596467.

Farmacopéia Brasileira (1988), 4th edn. Atheneu. São Paulo, Brazil.

Fraga HFS, Rosa PM, Morais AA, Pinto AC, Rezende CM (2006) Análise dos constituintes químicos do óleo essencial das folhas de Gallesia integrifolia (Sprengel) Harms (Phytolaccaceae). In: 29a Reunião Anual da Sociedade Brasileira de Química, Águas de Lindóia. 19 a 22 de maio de 2006.

Furtado FN, Silva VAR, Pereira JRG, Kisue A, Coelho FAS, Coelho MD (2013) In vitro assesment of Tagetes minuta essential oil potencial against Riphicephalus (Boophilus) microplus (Canestrini, 1887). Rev Biocienc. 19(1):104-110.

Gil ES (2007) Controle Físico-químico de qualidade de medicamentos, 2nd edn. Pharmabooks. São Paulo, Brazil.
Hansen J (1999) Inactivation of MXR1 abolishes formation of dimethyl sulfide from dimethyl sulfoxide in Saccharomyces cerevisiae. Appl Environ Microb. 65(9):3915-3919.

Hattula T, Granroth B (1974) Formation of dimethyl sulphide from S-methylmethionine in onion seedlings (Allium cepa). J Sci Food Agr. 25(12):1517-1521.

Kafle GM, Castro-Janer E, Mendes MC, Namindome A, Schumaker TTS (2012) Applicability of in vitro bioassays for the diagnosis of ivermectin resistance in Rhipicephalus microplus (Acari: Ixodidae). Vet Pathol. 184(2-4):212-220.

Kerudo A, Gonnot V, Ellong EN, Boyer L, Michel T, Adenet S, Rochefort K, Fernandez X (2015) Essential oil composition and biological activities of Petiveria alliacea L. from Martinique. J Essent Oil Res. 27(3):186-196.

Kyung KH, Lee YC (2001) Antimicrobial activities of sulfur compounds derived from S-alk (en) yl-L-cysteine sulfoxides in allium and brassica. Food Rev Int. 17(2):183198.

Landaud S, Helinck S, Bonnarme P (2008) Formation of volatile sulfur compounds and metabolism of methionine and other sulfur compounds in fermented food. Appl Microbiol Biot. 77(6):1191-1205.

Leite RC, Labruna PR, Oliveira AMF (1995) In vitro susceptibility of engorded females from different populations of Boophilus microplus to commercial acaricides. Rev Bras de Parasitol Vet. 4(2):283-284.

Lima HRP, Kaplan MAC, Cruz AVM (2003) Influence of abiotic factors on terpenoids production and variability in the plants. Floresta e Ambiente. 10(2):71-77.

Lomans BP, Van der Drift C, Pol A, Op den Camp HJM (2002) Microbial cycling of volatile organic sulfur compounds. Cell Mol Life Sci. 59(4):575-588.

Lorenzi H (2002) Árvores brasileiras: Manual de identificação e cultivo de plantas arbóreas nativas do Brasil, 4th edn. Instituto Plantarum de Estudos da Flora Ltda. São Paulo, Brazil.

Lyndon J, Lawrence WAD, Earle RV (1997) An insecticidal and acaricidal polysulfide metabolite from the roots of Petiveria alliacea. J Pestic Sci. 50(3):228-232.

Maia AJ, Schwan-Estrada KRF, Faria CMDR, Jardinetti VA, Botelho RV (2013) Bud break of 'Benitaka' grapevines treated with Gallesia integrifolia hydrolate. Rev Bras Frutic. 35(3):685-694.

Martins JR, Evans DE, Cereser VH, Corrêa BL (2002) Partial strategic tick control with in a herd of European breed cattle in the state of Rio Grande do Sul, Southern Brazil. Exp Appl Acarol. 27(3):241-251.

Moir M, Seaton JC, Suggett A (1980) 2,3,5-trithiahexane in the essential oil of Humulus lupulus. Phytochemistry. 19:2201.

Omolo MO, Okinyo D, Ndiege IO, Lwande W, Hassanali A (2004) Repellency of essential oils of some kenyan plants against Anopheles gambiae. Phytochemistry. 65(20):27972802.

Prates HT, Oliveira AB, Leite RC, Craveiro AA (1993) Antitick effect and chemical composition of the molasses grass essential oil. Pesq Agropec Bras. 28(5):621-625.

Reis PR, Rebelles PPR, Pereira MC, Liska GR, De Morais AR (2015) Effectiveness of sulfur applied to soil in controle of cicada Quesada gigas (Olivier) in coffee plant. Coffee Science. 10: 527-536.

Rosado-Aguillar JA, Aguilar-Caballero A, Rodriguez-Vivas RI, Borges-Argaez R, Garcia-Vazquez Z, MendezGonzalez M (2010) Acaricidal activity of extracts from Petiveria alliacea (Phytolaccaceae) against the cattle tick, 
Rhipicephalus (Boophilus) microplus (Acari: ixodidae). Vet Parasitol. 168(3-4):299-303.

Sambuichi RHR, Mielke MS, Pereira CE (2009) Lista de árvores nativas do sul da Bahia. In: Sambuichi RHR, Silva LAM, De Jesus MFC, Da Paixão JL Nossas árvores: conservação, uso e manejo de árvores nativas do sul da Bahia, 1st edn. Editus. Bahia, Brazil. 171-257.

Santos TRB, Castro NA, Bretanha LC, Schuch LFD, Freitag RA, Nizoli LQ (2015) Estudo in vitro da eficácia de citronela (Cymbopogon wybteruanus) sobre carrapato Rhipicephalus (Boophilus) microplus. Science Animal Health. 3(1):135-149.

Schünemann AP, Treptow R, Leite DL, Vendruscolo JL (2006) Pungency and chemical characteristics in bulbs of onion genotypes (Allium cepa L.) grown in Alto Vale do Itajaí, SC, Brasil. Rev Bras Agrocienc. 12(1):77-80.

Schutte L, Teranishi R (1974) Precursors of sulfur-containing flavor compounds. CRC Crit Rev Fd Technol. 4(4):457505.

Souza SAM, Meira MR, De Figueiredo LS, Martins ER (2010) Essential oils: the economic and sustainable. Rev Enc Biosfera. 6(10):1-11.
Silva Ferreira AC, Rodrigues P, Hoog T, De Pinho P G (2003) Influence of some technological parameters on the formation of dimethyl sulfide, 2-mercaptoethanol, methionol, and dimethyl sulfone in Portwines. J Agr Food Chem. 51(3):727-732.

Simões CM, Schenkel EP, Gosmann G, De Mello JCP, Mentz LA, Petrovick PR (2010) Farmacognosia da planta ao medicamento. In: Simões CM, Spitzer V. Óleos voláteis, 6th edn. Universidade/UFRGS/UFSC. Porto Alegre/Florianópolis, Brazil. 397-425.

Van Langenhove HJ, Cornelis CP, Schamp NM (1991) Identification of volatiles emitted during the blanching process of brussels sprouts and cauliflower. J Sci Food Agr. 55(3):483-487.

Vollhardt P, Schore N (2013) Química Orgânica: estrutura e função, 6th edn. Bookman. Porto Alegre, Brazil.

Winter A (2011) Química orgânica I para leigos, 1st edn. Alta Books. Rio de Janeiro, Brazil.

Yoshimura M, Nakano Y, Yamashita Y, Oho T, Saito T, Koga T (2000) Formation of methyl mercaptan from Lmethionine by Porphyromonas gingivalis. Infect Immun. 68(12):6912-6916. 